\title{
PRÉ-TRATAMENTO ALCALINO DO BAGAÇO DE CANA-DE- AÇÚCAR SOB CONDIÇÕES AMENAS DE PRESSÃO E TEMPERATURA: DIGESTIBILIDADE ENZIMÁTICA E AVALIAÇÃO DO POTENCIAL DO LICOR NEGRO OBTIDO
}

\author{
J. S. H. TANAKA; H. M. JESUS; S. S. SILVA; J. C. dos SANTOS* \\ Escola de Engenharia de Lorena, Universidade de São Paulo, Departamento de Biotecnologia \\ E-mail para contato: jsant200@usp.br
}

\begin{abstract}
RESUMO - O uso de materiais lignocelulósicos em bioprocessos depende da quebra de suas frações macromoleculares em compostos mais simples. Neste trabalho, avaliou-se o efeito do pré-tratamento do bagaço de cana-de-açúcar com $\mathrm{NaOH}$, a pressão atmosférica, sob diferentes condições de concentração de álcali e temperatura. $\mathrm{O}$ sólido obtido foi avaliado com relação a sua digestibilidade por preparado comercial de celulase, sendo avaliada a composição do licor resultante. Empregando $0,5 \mathrm{M}$ de $\mathrm{NaOH}$, tanto a $40^{\circ} \mathrm{C}$ quanto a $80^{\circ} \mathrm{C}$, observou-se um percentual de hidrólise da celulose cerca de 2,5 vezes superior em relação à matéria-prima não tratada, sendo obtidos, a partir do licor, entre 5-8 g/L de material rico em lignina e cerca de $1 \mathrm{~g} / \mathrm{L}$ de açúcares totais. Os resultados revelaram que a lignina foi $\mathrm{o}$ principal componente removido durante o pré-tratamento, resultando em um material mais rico em celulose. A digestibilidade enzimática da celulose foi superior no material pré-tratado em relação ao bagaço não tratado, sendo que o efeito da concentração de $\mathrm{NaOH}$ foi mais importante comparado ao da variável temperatura.
\end{abstract}

\section{INTRODUÇÃO}

Materiais lignocelulósicos são os principais constituintes da biomassa vegetal de resíduos e subprodutos agrícolas e florestais e representam uma fonte de carbono abundante e com elevado potencial para aplicação em processos biotecnológicos para obtenção de produtos de interesse. Estes materiais são frequentemente descartados sem aproveitamento de seu potencial, resultando ainda em problemas ambientais devido a seu acúmulo em grande quantidade (Kuhad e Sing, 1993; Cherubini, 2010).

A ampla gama de produtos que podem ser obtidos a partir desta biomassa levou ao conceito conhecido como "biorrefinaria" pois, fazendo-se uma analogia com as refinarias de petróleo, lignocelulósicos podem ser usados na obtenção de combustíveis e uma série de outros 


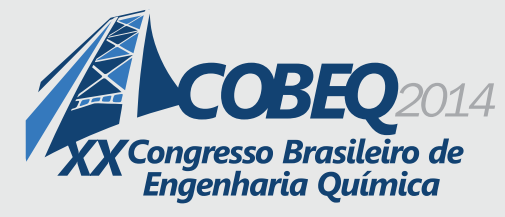

19 a 22 de outubro de 2014

Florianópolis/SC

compostos que podem ser utilizados como precursores químicos na cadeia produtiva ou diretamente na produção de alimentos e fármacos, entre outros (Cherubini, 2010). Entre estes usos, a produção de etanol combustível, chamado, neste caso, "de segunda geração", tem sido um dos temas de pesquisa mais destacados no mundo atual. Este destaque é devido a problemas ambientais devidos ao uso de combustíveis fósseis, os quais têm sido apontados como responsáveis por mudanças climáticas danosas devido ao acúmulo, na atmosfera, de gás carbônico oriundo de sua queima. Além disso, outros problemas como a instabilidade política em países produtores de petróleo, têm motivado a busca de substituição dos combustíveis fósseis por fontes renováveis, sendo o etanol uma alternativa interessante para uso em veículos automotores (Brethauer e Wyman, 2010; Sarkar et al., 2012).

O aproveitamento do potencial dos materiais lignocelulósicos em processos biotecnológicos, no entanto, depende do desenvolvimento de tecnologia viável para liberação de açúcares fermetescíveis a partir de suas frações macromoleculares. Estas, que incluem a celulose - constituída por unidades de glicose, a hemicelulose - constituída por unidades de diferentes açúcares, entre os quais pentoses como xilose e arabinose, e a lignina - macromolécula fenólica, formam uma estrutura fechada e de difícil acesso para agentes químicos ou biológicos (Fengel e Weneger, 1989; Agbor et al., 2011).

A celulose, principal constituinte da parede celular da biomassa vegetal, pode ser hidrolisada em moléculas de glicose pela ação de enzimas em um processo ambientalmente amigável sob condições brandas de temperatura e pressão. A glicose obtida pode então ser convertida em etanol pelo uso de leveduras. O processo enzimático, no entanto, requer uma etapa prévia de tratamento da matéria-prima para modificação da estrutura fechada do material, permitindo acesso às enzimas. Este pré-tratamento tem sido feito principalmente pelo uso de ácidos diluídos, em um processo sob condições de elevada temperatura e pressão, somando-se a isso as condições ácidas que exigem equipamento especial (Alvira et al., 2010).

Um alternativa que pode resultar em aumento da digestibilidade enzimática do material sob condições menos severas corresponde ao uso de pré-tratamento alcalino. Neste caso, álcalis como o hidróxido de sódio podem ser empregados sob pressão atmosférica até mesmo sob temperatura ambiente (Wu et al., 2011a; Wu et al., 2011b; Modenbach e Nokes, 2012). O pré-tratamento alcalino de lignocelulósicos resulta em um material sólido que pode ser submetido à hidrólise enzimática da celulose, mas também em um licor negro contendo lignina e hemicelulose solubilizadas (Xiao et al., 2001). Estes materiais podem ser utilizados em diferentes aplicações de interesse industrial, com potencial para obtenção de compostos diversos (Xiao et al., 2001; Mussato et al., 2007).

Neste contexto, o presente trabalho teve como objetivo avaliar o efeito do pré-tratamento alcalino do bagaço de cana-de-açúcar na digestibilidade da celulose por celulases comerciais, tendo sido também feita uma caracterização preliminar da composição do licor negro resultante do pré-tratamento, o que serve de base para avaliação de seu potencial de uso. 


\section{MATERIAIS E MÉTODOS}

\subsection{Materiais}

Enzima: foi utilizado preparado enzimático comercial Dyadic ${ }^{\circledR}$ Cellulase CP CONC (Dyadic International, Inc., Jupiter, Flórida, USA), o qual contém celulases e hemicelulases.

Matéria-prima e reagentes: o bagaço de cana-de-açúcar foi adquirido da Usina São Francisco (Sertãozinho, SP); todos os demais reagentes empregados foram em grau analítico.

\subsection{Metodologia}

Preparo e caracterização da matéria-prima: o bagaço de cana-de-açúcar foi seco ao sol até atingir umidade aproximada de 10\% conforme medido em balança de infravermelho Mark M163 (BEL Engineering, Piracicaba - SP), sendo então moído em um moinho de martelo marca Benedetti, modelo dupla 270 (Moinho Benedetti Ltda., Pinhal - SP). Em seguida, fez-se a análise granulométrica do material obtido utilizando um conjunto de peneiras padrão série Tyler montado em um agitador de peneiras adquirido da Bertel Indústrias Metalúrgicas Ltda. (Caieiras - SP). O bagaço foi caracterizado com relação a sua composição em extrativos, celulose, hemicelulose, lignina e cinzas de acordo com metodologia descrita por Masarin et al. (2011).

Pré-tratamento da biomassa: as amostras foram tratadas com solução aquosa de hidróxido de sódio em experimentos empregando diferentes valores de temperatura e concentração de álcali (Tabela 1). A relação mássica bagaço:meio reacional foi de 7,5\%, correspondendo a valores de relação mássica álcali: bagaço de 5 e $27 \%$ (tabela 1 ). O meio reacional contendo bagaço de solução alcalina foi colocado em frascos Erlenmeyer de $250 \mathrm{~mL}$ e mantidos durante $2 \mathrm{~h}$ sob agitação de $110 \mathrm{rpm}$ em um banho termostatizado Lindberg/Blue M SWB1122C-1 (Thermo Scientific, Asheville, NC, USA). Após reação, a solução foi filtrada a vácuo, sendo o sólido prétratado caracterizado e submetido à hidrólise enzimática da celulose. O licor filtrado foi submetido a procedimentos visando-se a sua caracterização composicional preliminar, conforme descrito a seguir.

Caracterização da biomassa pré-tratada: o sólido pré-tratado foi moído em um moinho de facas (Bodine Eletric Company, Chicago - EUA) até passar em uma peneira padrão série Tyler 20 MESH, sendo então caracterizado com relação a sua composição em celulose, hemicelulose e lignina, conforme descrito por Masarin et al. (2011).

Caracterização do licor negro: o licor negro obtido do pré-tratamento teve seu $\mathrm{pH}$ reduzido a 2,0 empregando solução $6 \mathrm{M}$ de ácido clorídrico, visando-se à precipitação de uma material rico em lignina. A suspensão obtida foi submetida à filtração a vácuo, sendo a massa seca de sólido obtida determinada após secagem em estufa a $105^{\circ} \mathrm{C}$. Uma alíquota do filtrado foi analisado com relação à concentração de lignina solúvel conforme descrito por Masarin et al. (2011). O restante do filtrado foi mantido sob refrigeração durante $24 \mathrm{~h}$, sendo em seguida centrifugado e filtrado a 
vácuo. O sólido obtido nestas etapas teve sua massa seca determinada após secagem em estufa a $105^{\circ} \mathrm{C}$, sendo esta massa somada à obtida anteriormente para o precipitado rico em lignina obtido após redução do pH. O filtrado foi analisado com relação ao teor de carboidratos pelo método fenol-sulfúrico, conforme Dubois et al. (1956).

Tabela 1 - Condições empregadas nos experimentos de pré-tratamento alcalino do bagaço de cana-de-açúcar

\begin{tabular}{|c|c|c|c|}
\hline \multirow[b]{2}{*}{ Experimento } & \multicolumn{3}{|c|}{ Condições } \\
\hline & $\begin{array}{c}\text { Temperatura } \\
\left({ }^{\circ} \mathrm{C}\right)\end{array}$ & $\begin{array}{r}\text { Concentração } \\
\text { de } \mathrm{NaOH}(\mathrm{M})\end{array}$ & $\begin{array}{c}\text { Relação } \\
\text { mássica } \\
\text { álcali: } \\
\text { bagaço } \\
(\%)\end{array}$ \\
\hline 1 & 40 & 0,1 & 5 \\
\hline 2 & 40 & 0,5 & 27 \\
\hline 3 & 80 & 0,1 & 5 \\
\hline 4 & 80 & 0,5 & 27 \\
\hline
\end{tabular}

Hidrólise Enzimática: a hidrólise do sólido obtido no pré-tratamento foi conduzida em frascos Erlenmeyer $50 \mathrm{~mL}$ contendo $15 \mathrm{~mL}$ de meio reacional constituído por suspensão contendo $2 \%$ de bagaço de cana-de-açúcar pré-tratado (massa seca) em tampão citrato $50 \mathrm{mM}$, além de preparado enzimático contendo celulase na proporção atividade enzimática: massa seca de bagaço de $10 \mathrm{FPU} / \mathrm{g}$. Os experimentos foram realizados em incubadora de movimento rotatório (Quimis Q816M20, Quimis Aparelhos Científicos, Diadema, SP) sob condições de 50 ${ }^{\circ} \mathrm{C}$ e $150 \mathrm{rpm}$ por 24 horas.

Análise da concentração de açúcares: A concentração de açúcares foi determinada empregando cromatografia liquida de alta eficiência (CLAE) com coluna BIO RAD AMINEX HPX 87p (300x7,8 mm), conforme Medina (2013).

Expressão dos resultados: os experimentos/análises foram realizados pelo menos em duplicata e os resultados expressos como valor médio \pm desvio padrão.

\section{RESULTADOS E DISCUSSÃO}

O bagaço de cana-de-açúcar seco e moído apresentou granulometria correspondente à passagem de mais de 90\% das partículas em uma peneira padrão Tyler $10 \mathrm{MESH}$ (abertura 1,65 $\mathrm{mm}$ ). Antes da moagem, apenas $45 \%$ das partículas passavam por esta peneira. A redução no tamanho das partículas favorece o pré-tratamento, aumentando a área superficial dos sólidos em contato com os reagentes. 
O bagaço moído foi então caracterizado com relação a sua composição, sendo obtidos os seguintes resultados, expressos em fração mássica (\%): celulose, 41,3 $\pm 2,1$; hemicelulose,

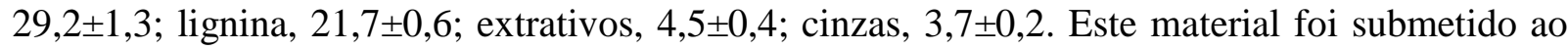
pré-tratamento alcalino, sendo a composição do sólido pré-tratado obtido mostrado, na Figura 1.

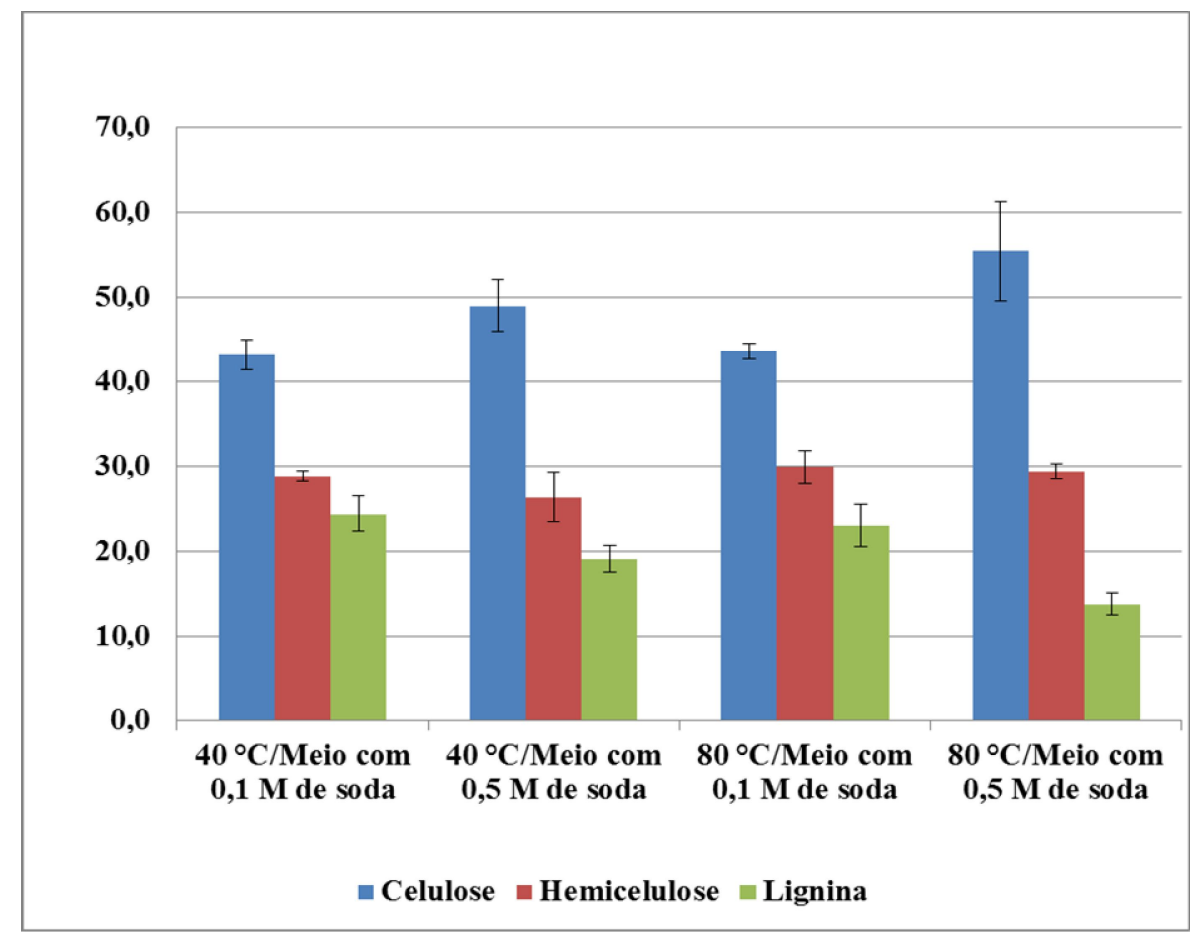

Figura 1 - Composição do bagaço de cana-de-açúcar pré-tratado empregando diferentes valores de concentração de álcalis e temperatura.

Conforme pode ser observado na figura, a utilização das condições mais amenas de concentração alcalina e temperatura resultou em um sólido pré-tratado com composição semelhante à da matéria-prima. $\mathrm{O}$ aumento tanto na concentração de $\mathrm{NaOH}$ quanto na temperatura, resultou em um material com menor quantidade de lignina e maior fração de celulose. Este comportamento pode ser explicado, considerando-se que pré-tratamentos alcalinos, diferentemente de processos ácidos ou hidrotérmicos, são mais efetivos na solubilização da lignina, apresentando menor efeito nos carboidratos (Carvalheiro et al., 2008). Esta afirmação, no entanto, embora corroborada pelos dados obtidos, é de caráter geral, uma vez que há relatos na literatura de remoção de frações carboidrato em presença de álcalis, em especial da hemicelulose. Como exemplo, cita-se o trabalho de Wu et al. (2011b), no qual bagaço de cana-de-açúcar foi submetido a pré-tratamento alcalino com hidróxido de sódio sob temperaturas de 25 e $50^{\circ} \mathrm{C}$, porém utilizando concentração de $\mathrm{NaOH}$ de $1-5 \mathrm{M}$. No presente trabalho, a redução na quantidade de lignina concomitantemente à manutenção na fração de hemicelulose pode ser indicativa de alguma remoção desta durante o pré-tratamento. 
A maior remoção de lignina em comparação aos carboidratos pôde ser verificada também pela análise preliminar realizada no licor negro obtido (Tabela 2). Esta análise, realizada visandose determinar o seu potencial de uso em aplicações de interesse, demonstrou que, nas condições experimentais avaliadas, o licor gerado possui maior quantidade de lignina em comparação ao teor de açúcares.

Tabela 2. Concentração de material rico em lignina e de açúcares totais no licor obtido após pré-tratamento do bagaço de cana-de-açúcar sob diferentes condições de concentração de álcali e temperatura.

\begin{tabular}{cccc}
\hline Experimento & Condições & Lignina no Licor $(\boldsymbol{g} / \mathbf{L})$ & Açúcares no Licor $(\boldsymbol{g} / \mathbf{L})$ \\
\hline 1 & $0,1 \mathrm{M} \mathrm{de} \mathrm{NaOH}, 40^{\circ} \mathrm{C}$ & 2,43 & 0,22 \\
2 & $0,5 \mathrm{M} \mathrm{de} \mathrm{NaOH}, 40^{\circ} \mathrm{C}$ & 8,62 & 1,18 \\
3 & $0,1 \mathrm{M} \mathrm{de} \mathrm{NaOH}, 80^{\circ} \mathrm{C}$ & 3,44 & 0,94 \\
4 & $0,5 \mathrm{M} \mathrm{de} \mathrm{NaOH}, 80^{\circ} \mathrm{C}$ & 5,1 & 1,17 \\
\hline
\end{tabular}

As mudanças composicionais, no entanto, não são o único fator determinante do aumento da digestibilidade enzimática do material submetido a pré-tratamento alcalino, sendo importante considerar fatores como mudanças em grau de polimerização dos constituintes do material, cristalinidade e porosidade (Carvalheiro et al., 2008). Assim, avaliou-se o efeito do prétratamento na digestibilidade enzimática do material sólido obtido. Assim, o material pré-tratado foi submetido a hidrólise enzimática com preparação comercial de celulase. A Tabela 3 mostra os resultados obtidos em comparação aos observados usando-se bagaço não tratado.

Tabela 3. Digestibilidade enzimática do bagaço de cana-de-açúcar não tratado ou submetido ao pré-tratamento alcalino sob diferentes condições de concentração de álcalis e temperatura.

\begin{tabular}{cc}
\hline Sistema/condições de pré-tratamento & Rendimento de hidrólise (\%) \\
\hline Bagaço não tratado & $11,0 \pm 2,4$ \\
Bagaço pré-tratado/ $0,1 \mathrm{M}$ de $\mathrm{NaOH}, 40^{\circ} \mathrm{C}$ & $15,5 \pm 0,6$ \\
Bagaço pré-tratado $/ 0,5 \mathrm{M}$ de $\mathrm{NaOH}, 40^{\circ} \mathrm{C}$ & $27,6 \pm 0,5$ \\
Bagaço pré-tratado $/ 0,1 \mathrm{M}$ de $\mathrm{NaOH}, 80^{\circ} \mathrm{C}$ & $16,5 \pm 0,2$ \\
Bagaço pré-tratado/ $0,5 \mathrm{M}$ de $\mathrm{NaOH}, 80^{\circ} \mathrm{C}$ & $21,4 \pm 0,2$ \\
\hline
\end{tabular}

Conforme mostrado na Tabela 3, o pré-tratamento resultou em maior rendimento de 


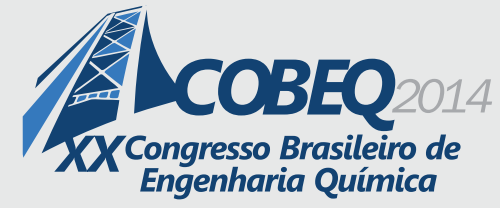

hidrólise, em especial quando maior concentração de $\mathrm{NaOH}$ foi empregada. De fato, pode-se observar que o efeito da concentração de $\mathrm{NaOH}$ foi mais importante para o aumento na digestibilidade enzimática da celulose presente no material em comparação ao efeito da temperatura. Mesmo a $40^{\circ} \mathrm{C}$, o rendimento de hidrólise após $24 \mathrm{~h}$ de processo enzimático foi cerca de 2,5 vezes superior ao obtido com o material não tratado.

\section{CONCLUSÕES}

O trabalho apresentado teve como objetivo avaliar o efeito do pré-tratamento alcalino do bagaço de cana-de-açúcar na digestibilidade da celulose por preparação comercial de celulase, tendo sido também feita uma caracterização preliminar da composição do licor negro resultante do pré-tratamento, o que poderá servir de base experimentos futuros visando-se ao seu uso em aplicações de interesse. Os resultados revelaram que a lignina foi o principal componente removido durante o pré-tratamento, resultando em um material mais rico em celulose. A digestibilidade enzimática da celulose foi superior no material pré-tratado em relação ao bagaço não tratado, sendo que o efeito da concentração de $\mathrm{NaOH}$ foi mais importante comparado ao da variável temperatura.

\section{AGRADECIMENTOS}

Os autores agradecem ao suporte financeiro da Universidade de São Paulo.

\section{REFERÊNCIAS}

AGBOR, V. B.; CICEK, N.; SPARLING, R.; BERLIN, A.; LEVIN, D. B. Biomass pretreatment: Fundamentals toward application. Biotechnology Advances, 29, 675-685, 2011.

ALVIRA, P.; TOMÁS-PEJÓ, E.; BALLESTEROS, M.; NEGRO, M. J. Pretreatment technologies for an efficient bioethanol production process based on enzymatic hydrolysis: A review. Bioresource Technology, 101, 4581-4861, 2010.

BRETHAUER, S.; WYMAN, C. E. Review: Continuous hydrolysis and fermentation for cellulosic ethanol production. Bioresource Technology, 101, 4862-4874, 2010.

CARVALHEIRO F., DUARTE L. C.; GÍRIO F.M.: Hemicellulose biorefineries: a review on biomass pretreatments. Journal of Scientific \& Industrial Research, 67, 849-864, 2008.

CHERUBINI, F. The biorefinery concept: Using biomass instead of oil for producing energy and chemicals. Energy Conversion and Management, 51, 1412-1421, 2010.

DUBOIS, M.; GILLES, K. A.; HAMILTON, J. K.; REBERS, P. A.; SMITH, F. Colorimetric Method form Determination of Sugars and Related Substaces. Nature, v. 28, n. 3, $350-356,1956$. 
FENGEL, D., WEGENER, G. Wood Chemistry, Ultrastructure, Reactions. Berlin: Walter de Gruyter, 613 p., 1989.

KUHAD, R. C., SINGH, A. Lignocellulose Biotechnology: Current and Future Prospects. Critical Reviews in Biotechnology, 13, 151-73, 1993.

MASARIN, F.; GURPILHARES, D. B.; BAFFA. D. C. F.; BARBOSA, M. H. P.; CARVALHO, W.; FERRAZ, A.; MILAGRES, A. M. F. Chemical composition and enzymatic digestibility of sugarcane clones selected for varied lignin content. Biotechnology for Biofuels. 4 : $55,2011$.

MEDINA, K. J. D. . Lorena/SP: Universidade de São Paulo, Escola de Engenharia de Lorena, 2013, (Tese de Doutorado), 172 p.

MODENBACH, A.; NOKES, S. E. The Use of High-Solids Loadings in Biomass Pretreatment-A Review. Biotechnology and Bioengineering, 109 (6), 2012.

MUSSATO, S. I.; FERNANDES, F.;ROBERTO, I. C. Lignin recovery from brewer's spent grain black liquor. Carbohydrate Polymers, 70, 218-223, 2007.

SARKAR, N.; GHOSH, S. K.; BANNERJEE, S.; AIKAT, K. Bioethanol production from agricultural wastes: An overview. Renewable Energy, 37, 19-27, 2012.

WU, L.; ARAKANE, M.; IKE, M.; WADA, M.; TAKAI, T.; GAU, M.; TOKUYASU, K. Low temperature alkali pretreatment for improving enzymatic digestibility of sweet sorghum bagasse for ethanol production. Bioresource Technology, 102, 4793-4799, $2011 \mathrm{a}$.

WU, L.; Li, Y.; ARAKANE, M.; IKE, M.; WADA, M.; TERAJIMA, Y.; ISHIKAWA, S.; TOKUYASU, K. Efficient conversion of sugarcane stalks into ethanol employing low temperature alkali pretreatment method. Bioresource Technology, 102, 11183-11188, $2011 \mathrm{~b}$.

XIAO, B.; SUN, X. F.; SUN, R. Chemical, structural, and thermal characterizations of alkali-soluble lignins and hemicelluloses, and cellulose from maize stems, rye straw, and rice straw. Polymer Degradation and Stability, 74, 307-319, 2001. 